\title{
ON THE STRUCTURE OF FLOWS THROUGH PIPE-LIKE DOMAINS SATISFYING A GEOMETRICAL CONSTRAINT
}

Abstract. We study solutions of the steady Navier-Stokes equations in a bounded 2D domain with the slip boundary conditions admitting flow across the boundary. We show conditions guaranteeing uniqueness of the solution. Next, we examine the structure of the solution considering an approximation given by a natural linearization. Suitable error estimates are also obtained.

1. Introduction. In this note we analyze a model of two-dimensional flows of viscous incompressible fluids through bounded domains with nontrivial flows across the boundary. The motion is governed by the steady Navier-Stokes equations

$$
\begin{array}{ll}
v \cdot \nabla v-\nu \Delta v+\nabla p=F & \text { in } \Omega, \\
\operatorname{div} v=0 & \text { in } \Omega,
\end{array}
$$

where $v=\left(v^{1}, v^{2}\right)$ is the velocity of the fluid, $p$ the pressure, $\nu$ the constant positive viscous coefficient, $F$ the external force, and $\Omega$ a simply connected domain where our motion is studied.

We supplement (1.1) with the slip boundary conditions involving friction

$$
\begin{array}{ll}
n \cdot \mathbf{T}(v, p) \cdot \tau+f v \cdot \tau=0 & \text { on } \partial \Omega, \\
n \cdot v=d & \text { on } \partial \Omega,
\end{array}
$$

where $n$ and $\tau$ are the normal and tangent vectors to $\partial \Omega ; f$ describes the friction between the fluid and the boundary, it is nonnegative, in general

2000 Mathematics Subject Classification: 35Q30, 75D05.

Key words and phrases: Navier-Stokes equations, uniqueness, structure of solutions, geometrical constraint.

Supported by Polish KBN grant 2 PO3A 00223. 
nonconstant; and $\mathbf{T}(\cdot, \cdot)$ is the stress tensor of Newtonian fluids, i.e.

$$
\mathbf{T}(v, p)=\nu \mathbf{D}(v)-p \operatorname{Id}=\left\{\nu\left(v_{, j}^{i}+v_{, i}^{j}\right)-p \delta_{i j}\right\}_{i, j=1,2} .
$$

The function $d$ describes the inflow and outflow across the boundary.

This type of boundary conditions may be treated as an alternative to the standard Dirichlet boundary data

$$
u=D \quad \text { on } \partial \Omega .
$$

The existing theory for problem (1.1), (1.4) in general domains for nonzero data $D$ yields existence of solutions only for small magnitudes of fluxes given by boundary data. For arbitrarily large data we are able to find solutions only for problems in simply connected domains [3, 4, Chap. VIII, 6]. The difficulty is connected with applications of the energy method which seems to be a natural way for the Dirichlet problem. In this approach there is a need to construct an extension of the boundary data, say $\mathcal{D}$, such that

$$
\operatorname{div} \mathcal{D}=0,\left.\quad \mathcal{D}\right|_{\partial \Omega}=D .
$$

Moreover, the greatest obstacle to applying the energy method comes from the requirement that the field $\mathcal{D}$ must satisfy the estimate

$$
\left|\int_{\Omega} u \cdot \nabla \mathcal{D} u d x\right| \leq \delta\|u\|_{H_{0}^{1}(\Omega)}
$$

for a small $\delta$ and for all $u \in H_{0}^{1}(\Omega)$. The smallness of $\delta$ is determined by the magnitude of the viscosity. For arbitrary $D$ we can find the field $\mathcal{D}$ only for a simply connected domain.

To see the crucial role of inequality (1.6) we modify (1.1) by decomposing the velocity as

$$
v=\mathcal{D}+u
$$

Then from (1.1) we get

$$
\begin{array}{ll}
v \cdot \nabla u-\nu \Delta u+\nabla p=F-u \cdot \nabla \mathcal{D}-\mathcal{D} \cdot \nabla \mathcal{D}+\nu \Delta \mathcal{D} & \text { in } \Omega, \\
\operatorname{div} u=0 & \text { in } \Omega .
\end{array}
$$

By (1.7) the new unknown function has good properties at the boundary, i.e.

$$
\left.u\right|_{\partial \Omega}=0 .
$$

To obtain an energy estimate for (1.8) we need to bound the second term of the r.h.s. of $(1.8)_{1}$. Here we apply (1.6) remembering that $\Omega$ is simply connected and the field $\mathcal{D}$ can be constructed.

The uniqueness of solutions for the Dirichlet problem is ensured only for small $D$. This is a consequence of the energy approach which does not involve the geometrical properties of the domain $\Omega$. 
The inadequacy of the method for the Dirichlet problem (1.1), (1.4) follows from the modification of the original system. Due to the general properties of the energy technique we need to have homogeneous boundary data, so we introduce the field $\mathcal{D}$ and consider the function $u$ instead of $v$. But this change leads to the appearance of a bad term $-u \cdot \nabla \mathcal{D}$ in equation $(1.8)_{1}$ which is a consequence of subtraction. The system loses its physical structure.

That is the motivation for a new approach to this issue. The technique should be based on the original equations and we have to avoid any subtractions for nonlinear systems. In this paper we present such a technique, but only for the slip boundary conditions (1.2). Moreover we will have to assume an extra property of the domain and smallness of the effective friction $f / \nu$. But as a result we obtain an a priori estimate for the solutions to problem (1.1), (1.2) without any bound on the boundary data $d$ and external force $F$. The restriction on $\Omega$ is completely independent of the magnitude of the flow. And the a priori bound has a linear form just as the standard energy estimate for homogeneous boundary data.

Our approach takes into account the geometrical features of the domain, hence the uniqueness conditions will depend on the properties of $\Omega$, not only on the magnitude of the data.

A key idea is a reformulation of problem (1.1), (1.2). A crucial role will be played by the vorticity of the velocity,

$$
\alpha=\operatorname{rot} v=v_{, 1}^{2}-v_{, 2}^{1} .
$$

Note that our method works only in two space dimensions, since the vorticity is then a scalar function and in three dimensions it is a vector.

Taking the rotation of $(1.1)_{1}$ we obtain a scalar equation

$$
v \cdot \nabla \alpha-\nu \Delta \alpha=\operatorname{rot} F \quad \text { in } \Omega .
$$

This follows from the fact that

$$
\operatorname{rot}(v \cdot \nabla v)=v \cdot \nabla \alpha,
$$

but only in our case, since in general $3-\mathrm{D}$ we have

$$
\operatorname{rot}(v \cdot \nabla v)=v \cdot \nabla \operatorname{rot} v-\operatorname{rot} v \cdot \nabla v .
$$

We need to add a boundary condition to (1.11). Here we apply an interesting feature of the slip boundary conditions. From (1.2) we compute the Dirichlet datum for the vorticity as follows:

$$
\alpha=(2 \chi-f / \nu) v \cdot \tau-2 d_{, s} \quad \text { on } \partial \Omega,
$$

where $\chi$ is the curvature of $\Omega$ and $s$ is the arc length parameter on $\partial \Omega$. 
To close system (1.11), (1.14) we add the following elliptic problem for the velocity:

$$
\begin{array}{ll}
\operatorname{rot} v=\alpha & \text { in } \Omega, \\
\operatorname{div} v=0 & \text { in } \Omega, \\
n \cdot v=d & \text { on } \partial \Omega .
\end{array}
$$

It is obvious that the coupled system (1.11), (1.14), (1.15) is equivalent to the original one (1.1), (1.2). It will turn out to be more suitable for our analysis.

The main advantage of this approach is the a priori estimate. Problem (1.11), (1.14) admits a sort of maximum principle.

Multiply (1.11) by

$$
\left(\alpha-k^{*}\right)_{+}=\max \left\{\alpha-k^{*}, 0\right\},
$$

where

$$
k^{*}=\sup _{x \in \partial \Omega}(2 \chi-f / \nu) v \cdot \tau-2 d_{, s} .
$$

By definition (1.16) the function $\left(\alpha-k^{*}\right)_{+}$is zero on the boundary, hence

$$
\int_{\Omega} \frac{1}{2} v \cdot \nabla\left(\alpha-k^{*}\right)_{+} d x+\nu \int_{\Omega}\left|\nabla\left(\alpha-k^{*}\right)_{+}\right|^{2} d x=-\int_{\Omega} F \cdot \nabla^{\perp}\left(\alpha-k^{*}\right)_{+},
$$

which implies

$$
\left\|\nabla\left(\alpha-k^{*}\right)_{+}\right\|_{L_{2}(\Omega)} \leq \frac{1}{\nu}\|F\|_{L_{2}(\Omega)} .
$$

The same bound is obtained for

$$
\left(\alpha-k_{*}\right)_{-}=\min \left\{\alpha-k_{*}, 0\right\}
$$

with

$$
k_{*}=\inf _{x \in \partial \Omega}(2 \chi-f / \nu) v \cdot \tau-2 d_{, s}
$$

i.e.

$$
\left\|\nabla\left(\alpha-k_{*}\right)_{-}\right\|_{L_{2}(\Omega)} \leq \frac{1}{\nu}\|F\|_{L_{2}(\Omega)} .
$$

This leads to the following decomposition of the vorticity:

$$
\alpha=\alpha_{L_{\infty}}+\alpha_{H_{0}^{1}},
$$

where

$$
\begin{aligned}
\alpha_{L_{\infty}} \in L_{\infty}(\Omega), & \left\|\alpha_{L_{\infty}}\right\|_{L_{\infty}(\Omega)} \leq \max \left\{k^{*}, k_{*}\right\}, \\
\alpha_{H_{0}^{1}} \in H_{0}^{1}(\Omega), \quad\left\|\nabla \alpha_{H_{0}^{1}}\right\|_{L_{2}(\Omega)} & \leq \frac{1}{\nu}\|F\|_{L_{2}(\Omega)} .
\end{aligned}
$$


This way we obtain an $L_{\infty}(\Omega)+H_{0}^{1}(\Omega)$ estimate for $\alpha$ :

$$
\begin{aligned}
\|\alpha\|_{L_{\infty}(\Omega)+H_{0}^{1}(\Omega)} \leq & \|2 \chi-f / \nu\|_{L_{\infty}(\partial \Omega)}\|v\|_{C(\partial \Omega)} \\
& +c\|d\|_{W_{\infty}^{1}(\partial \Omega)}+\frac{1}{\nu}\|F\|_{L_{2}(\Omega)} .
\end{aligned}
$$

which follows from (1.24) and (1.25).

Next, the standard theory of elliptic operators yields the following bound for the solutions of (1.15):

$$
\|v\|_{C(\Omega)} \leq A(\Omega)\|\alpha\|_{L_{\infty}(\Omega)+H_{0}^{1}(\Omega)}+c\|d\|_{W_{\infty}^{1}(\Omega)} .
$$

Estimates (1.26) and (1.27) lead to the main a priori bound

$$
\begin{aligned}
& \|v\|_{C(\Omega)}+\|\alpha\|_{L_{\infty}(\Omega)+H_{0}^{1}(\Omega)} \\
& \quad \leq B\left(1-A(\Omega)\|2 \chi-f / \nu\|_{L_{\infty}(\partial \Omega)}\right)^{-1}\left(\|d\|_{W_{\infty}^{1}(\Omega)}+\nu^{-1}\|F\|_{L_{2}(\Omega)}\right),
\end{aligned}
$$

provided the following condition is valid:

$$
G C=A(\Omega)\|2 \chi-f / \nu\|_{L_{\infty}(\partial \Omega)}<1 .
$$

The above constraint is a geometrical restriction on the shape of the domain. Since $f / \nu$ is nonnegative and may be chosen independently of the domain, to satisfy (1.29) we only need to control the negative part of the curvature. Hence the domain must satisfy

$$
A(\Omega) \inf _{x \in \partial \Omega} \min \{\chi, 0\}>-1 .
$$

Then we are able to find $f$ and $\nu$ such that (1.29) is satisfied.

The constant $A(\Omega)$ is optimal and depends only on the geometrical features of $\Omega$. In general $A(\Omega) \sim H$, where

$$
\begin{gathered}
H=\min _{\phi \in[0, \pi)} h_{\phi}, \\
h_{\phi}=\max _{y_{1} \in \mathbb{R}} \lambda_{1}\left\{\left(y_{1}, y_{2}\right) \in \Omega: y_{2} \in \mathbb{R}\right\},
\end{gathered}
$$

where $\lambda_{1}$ is the one-dimensional Lebesgue measure and $y$ is the coordinate system obtained from the original $x$-coordinates by rotation through angle $\phi$.

The bound obtained yields the following existence result.

Theorem A. Let $0<a<1, F \in L_{2}(\Omega)$ and $d \in W_{\infty}^{1}(\partial \Omega)$. If

$$
A(\Omega)\|2 \chi-f / \nu\|_{L_{\infty}(\partial \Omega)}<1,
$$

then there exists at least one solution to problem (1.1), (1.2) such that

$$
v \in C^{a}(\Omega), \quad \operatorname{rot} v \in L_{\infty}(\Omega)+H_{0}^{1}(\Omega),
$$

and the following estimate is valid:

$$
\begin{aligned}
\|v\|_{C^{a}(\Omega)}+\left\|(\operatorname{rot} v)_{L_{\infty}}\right\|_{L_{\infty}(\Omega)}+\left\|(\operatorname{rot} v)_{H_{0}^{1}}\right\|_{H_{0}^{1}(\Omega)} \\
\quad \leq B\left(\|d\|_{W_{\infty}^{1}(\partial \Omega)}+\nu^{-1}\|F\|_{L_{2}(\Omega)}\right)=S_{0},
\end{aligned}
$$


where $\operatorname{rot} v=(\operatorname{rot} v)_{L_{\infty}}+(\operatorname{rot} v)_{H_{0}^{1}},(\operatorname{rot} v)_{L_{\infty}} \in L_{\infty}(\Omega),(\operatorname{rot} v)_{H_{0}^{1}} \in H_{0}^{1}(\Omega)$ and $B$ is independent of $\nu$.

We skip the proof of Theorem A, since it follows from the a priori bound (1.28) proved at the beginning. The resulting regularity of the solutions guarantees existence by a standard application of the Leray-Schauder fixed point theorem. A similar proof can be found in [5, Theorem 3.1].

As an alternative to this assumption, for bounded $d$, we may assume the existence of a vector field $\mathcal{D}$ such that

$$
\begin{gathered}
\left.n \cdot \mathcal{D}\right|_{\partial \Omega}=d, \quad \operatorname{div} \mathcal{D}=0, \quad \operatorname{rot} \mathcal{D}=0, \\
\mathcal{D} \in W_{\infty}^{1}(\Omega) \cap W_{\infty}^{1}(\partial \Omega) .
\end{gathered}
$$

The construction of such a field is given in Section 4 .

One of the aims of the present note is to find conditions which imply uniqueness of the solutions.

In Section 3 we prove the following theorem.

THEOREM B. Let the assumptions of Theorem $\mathrm{A}$ be satisfied and suppose that either

$$
A(\Omega)\left(\frac{S_{0}}{\nu}\left(|\Omega|^{1 / 2}+H\right)+\|2 \chi-f / \nu\|_{L_{\infty}(\partial \Omega)}\right)<1
$$

or

$$
\frac{B_{2}^{2}(1+H)^{2}}{\nu}\left(B_{1}^{2}\|d\|_{L_{\infty}(\partial \Omega)}+S_{0}\right)<1 .
$$

Then the system (1.1)-(1.2) admits only one solution.

A comparison of these two conditions shows that the standard energy approach neglects the influence of the features of the domain. (1.39) implies that $S_{0}$ and $\|d\|_{L_{\infty}}$ have to be small. Condition (1.38) takes into account the quantities $h$ and $|\Omega|$ as well as condition (1.33) and admits large data (provided $H$ and $|\Omega|$ are small).

A natural consequence of the uniqueness result is an analysis of the structure of solutions. Since their norms are relatively small we want to find their series expansion. In Section 4 we prove the following result.

TheOREm C. Let $S_{0}$ be sufficiently small. Then the solutions of problem (1.1)-(1.2) have the form

$$
v=u^{0}+u^{1}+\cdots+u^{N}+u
$$

for any $N \in \mathbb{N}$, where $u^{l}$ is a solution of a linear system which depends only on $u^{l-1}, \ldots, u^{0}$ and the data; moreover

$$
\left\|u^{l}\right\|_{C^{a}(\Omega)} \leq \Xi^{l}
$$


for $l=0, \ldots, N$, and

$$
\|u\|_{C^{a}(\Omega)} \leq B \Xi^{N+1},
$$

where $\Xi=B_{0} S_{0}$ and $B_{0}$ depends on the constants of the problem.

Corollary. The solution given by Theorem $C$ can be expressed by the following series:

$$
v=\sum_{l=0}^{\infty} u^{l}
$$

This follows from the estimates of Theorem $\mathrm{C}$ and the fact that $\Xi<1$.

The key element of Theorem $\mathrm{C}$ is the bound (1.41). Our method makes it possible to obtain information in Hölder class. Of course, this can be done, since we already have Theorem A with bound (1.31). Thus, Theorem $\mathrm{C}$ is a consequence of Theorems A and B. Applying the standard energy approach we would obtain a weaker result with poorer information about the approximation error.

2. Notation. Throughout the paper we use the standard notation [7]. By $L_{p}(\Omega)$ we denote the Lebesgue space of $p$-integrable functions with the norm

$$
\begin{gathered}
\|f\|_{L_{p}(\Omega)}=\left(\int_{\Omega}|f(x)|^{p} d x\right)^{1 / p} \quad \text { for } 1 \leq p<\infty, \\
\|f\|_{L_{\infty}(\Omega)}=\underset{x \in \Omega}{\operatorname{essiup}}|f(x)| \quad \text { for } p=\infty .
\end{gathered}
$$

By $C^{a}(\Omega)$ we denote the space of Hölder continuous functions with the norm

$$
\|f\|_{C^{a}(\Omega)}=\sup _{x \in \Omega}|f(x)|+\sup _{x, y \in \Omega ; x \neq y} \frac{|f(x)-f(y)|}{|x-y|^{a}} .
$$

Moreover $X+Y$, for $X, Y$ Banach spaces, denotes the space

$$
X+Y=\{z=x+y: x \in X \text { and } y \in Y\}
$$

with the norm

$$
\|z\|_{X+Y}=\inf \left\{\|x\|_{X}+\|y\|_{Y}: x+y=z\right\} .
$$

The following auxiliary results are well known $[1,2,7]$.

Lemma 2.1. Let $\partial \Omega \in C^{2}$ and $u \in W_{p}^{1}(\Omega)$. Then the trace

$$
\widetilde{u}=\left.u\right|_{\partial \Omega}
$$

is well defined as a function in $W_{p}^{1-1 / p}(\partial \Omega)$ and

$$
\|\widetilde{u}\|_{W_{p}^{1-1 / p}(\partial \Omega)} \leq c\|u\|_{W_{p}^{1}(\Omega)} .
$$


Lemma 2.2 (Korn inequality). Let $u \in H^{1}(\Omega)$ with $\operatorname{div} u=0$ and $\left.n \cdot u\right|_{\partial \Omega}=0$. Then

$$
\|u\|_{H^{1}(\Omega)} \leq c\|\mathbf{D}(u)\|_{L_{2}(\Omega)} .
$$

Lemma 2.3. Let $b \in W_{p}^{2-1 / p}(\partial \Omega)$. Then the solution of the problem

$$
\begin{array}{ll}
\Delta \varphi=0 & \text { in } \Omega, \\
\varphi=b & \text { on } \partial \Omega
\end{array}
$$

satisfies

$$
\|\varphi\|_{W_{p}^{2}(\Omega)} \leq c\|b\|_{W_{p}^{2-1 / p}(\partial \Omega)} .
$$

3. Proof of Theorem B. In this section we prove Theorem B. The two conditions (1.38) and (1.39) follow from two different approaches to the uniqueness issue. The first is based on the analysis of the coupled system (1.11), (1.14), (1.15).

Take two solutions of problem (1.1), say $v^{1}$ and $v^{2}$. They satisfy

$$
\begin{array}{ll}
v^{i} \cdot \nabla \alpha^{i}-\nu \Delta \alpha^{i}=\operatorname{rot} F & \text { in } \Omega, \\
\alpha^{i}=(2 \chi-f / \nu) v^{i} \cdot \tau-2 d_{, s} & \text { on } \partial \Omega, \\
\operatorname{rot} v^{i}=\alpha^{i} & \text { in } \Omega, \\
\operatorname{div} v^{i}=0 & \text { in } \Omega, \\
n \cdot v^{i}=d & \text { on } \partial \Omega,
\end{array}
$$

for $i=1,2$. Set

$$
V=v_{1}-v_{2}, \quad R=\alpha_{1}-\alpha_{2} .
$$

Subtracting (3.1) for $i=1$ and $i=2$ we get

$$
\begin{array}{ll}
v^{1} \cdot \nabla R-\nu \Delta R=-V \cdot \nabla \alpha^{2} & \text { in } \Omega, \\
R=(2 \chi-f / \nu) V \cdot \tau & \text { on } \partial \Omega, \\
\operatorname{rot} V=R & \text { in } \Omega, \\
\operatorname{div} V=0 & \text { in } \Omega, \\
n \cdot V=0 & \text { on } \partial \Omega .
\end{array}
$$

We repeat the estimations (1.16)-(1.28) to obtain a condition controlling the uniqueness. Let

$$
K^{*}=\sup _{x \in \partial \Omega}(2 \chi-f / \nu) V \cdot \tau
$$

Multiply (3.3) 1 by

$$
\left(R-K^{*}\right)_{+}=\max \left\{R-K^{*}, 0\right\},
$$


then integrate over $\Omega$ to get

$$
\nu \int_{\Omega}\left|\nabla\left(R-K^{*}\right)_{+}\right|^{2} d x \leq\left|\int_{\Omega} V \cdot \nabla \alpha^{2}\left(R-K^{*}\right)_{+} d x\right| .
$$

By Theorem A we know that

$$
\alpha^{2}=\alpha_{L_{\infty}}^{2}+\alpha_{H_{0}^{1}}^{2},
$$

where $\alpha_{L_{\infty}}^{2} \in L_{\infty}(\Omega)$ and $\alpha_{H_{0}^{1}}^{2} \in H_{0}^{1}(\Omega)$. Then

$$
\begin{aligned}
\int_{\Omega} V & \cdot \nabla \alpha^{2}\left(R-K^{*}\right)_{+} d x \\
& =-\int_{\Omega} V \cdot \nabla\left(R-K^{*}\right)_{+} \alpha_{L_{\infty}}^{2} d x+\int_{\Omega} V \cdot \nabla \alpha_{H_{0}^{1}}^{2}\left(R-K^{*}\right)_{+} d x .
\end{aligned}
$$

The first term is bounded as follows:

$$
\begin{aligned}
\left\|V \alpha_{L_{\infty}}^{2}\right\|_{L_{2}(\Omega)} \| \nabla(R- & \left.K^{*}\right)_{+} \|_{L_{2}(\Omega)} \\
& \leq S_{0}|\Omega|^{1 / 2}\|V\|_{L_{\infty}(\Omega)}\left\|\nabla\left(R-K^{*}\right)_{+}\right\|_{L_{2}(\Omega)} .
\end{aligned}
$$

The second is handled in the following way:

$$
\begin{aligned}
\left\|V \cdot \nabla \alpha_{H_{0}^{1}}^{2}\right\|_{L_{2}(\Omega)}\left\|\left(R-K^{*}\right)_{+}\right\|_{L_{2}(\Omega)} \\
\leq S_{0} H\|V\|_{L_{\infty}(\Omega)}\left\|\nabla\left(R-K^{*}\right)_{+}\right\|_{L_{2}(\Omega)} .
\end{aligned}
$$

Thus, we get

$$
\left\|\nabla\left(R-K^{*}\right)_{+}\right\|_{L_{2}(\Omega)} \leq \frac{S_{0}}{\nu}\left(|\Omega|^{1 / 2}+H\right)\|V\|_{L_{\infty}(\Omega)} .
$$

The same bound is obtained for

$$
\left(R-K_{*}\right)_{-}=\min \left\{R-K_{*}, 0\right\}
$$

with

$$
K_{*}=\inf _{x \in \partial \Omega}(2 \chi-f / \nu) V \cdot \tau .
$$

Thus

$$
\left\|\nabla\left(R-K_{*}\right)_{-}\right\|_{L_{2}(\Omega)} \leq \frac{S_{0}}{\nu}\left(|\Omega|^{1 / 2}+H\right)\|V\|_{L_{\infty}(\Omega)} .
$$

Next, we find an estimate for the solution of $(3.3)_{3,4,5}$, analogous to $(1.27)$ :

$$
\|V\|_{C(\Omega)} \leq A(\Omega)
$$

$\cdot\left(\left\|\nabla\left(R-K_{*}\right)_{-}\right\|_{L_{2}(\Omega)}+\left\|\nabla\left(R-K^{*}\right)_{+}\right\|_{L_{2}(\Omega)}+\max \left\{\left|K^{*}\right|,\left|K_{*}\right|\right\}\right)\|V\|_{C(\Omega)}$. Applying (3.4), (3.11), (3.13) and (3.14), we get

$$
\|V\|_{C(\Omega)} \leq A(\Omega)\left(\frac{S_{0}}{\nu}\left(|\Omega|^{1 / 2}+H\right)+\|2 \chi-f / \nu\|_{L_{\infty}(\partial \Omega)}\right)\|V\|_{C(\Omega)} .
$$


Thus $V$ is zero if

$$
A(\Omega)\left(\frac{S_{0}}{\nu}\left(|\Omega|^{1 / 2}+H\right)+\|2 \chi-f / \nu\|_{L_{\infty}(\partial \Omega)}\right)<1,
$$

which is (1.38).

Note that the above condition depends on $|\Omega|$. It is possible to remove this dependence by localization of problem (3.3). The next result provides different information, independent of $|\Omega|$. It follows by applying the standard energy method.

Using the same notation as for (3.3), from (1.1) and (1.2) we get

$$
\begin{array}{ll}
v^{1} \cdot \nabla V-\nu \Delta V+\nabla P=-V \cdot \nabla v^{2} & \text { in } \Omega, \\
\operatorname{div} V=0 & \text { in } \Omega, \\
n \cdot \mathbf{T}(V, P) \cdot \tau+f V \cdot \tau=0 & \text { on } \partial \Omega, \\
n \cdot V=0 & \text { on } \partial \Omega .
\end{array}
$$

Multiplying (3.18) ${ }_{1}$ by $V$, remembering that

$$
-\nu \Delta V+\nabla P=-\operatorname{div} \mathbf{T}(V, P),
$$

we get

$$
\int_{\Omega} v^{1} \nabla V V d x+\nu \int_{\Omega}|\mathbf{D}(V)|^{2} d x+\int_{\partial \Omega} f(V \cdot \tau)^{2} d \sigma=-\int_{\Omega} V \cdot \nabla v^{2} V d x,
$$

hence

$$
\nu \int_{\Omega}|\mathbf{D}(V)|^{2} d x+\int_{\partial \Omega} f(V \cdot \tau)^{2} d \sigma \leq \int_{\partial \Omega}|d||V|^{2} d \sigma+\int_{\Omega}\left|v^{2}\right||V||\nabla V| d x .
$$

The first term of the r.h.s. of (3.21) can be estimated as follows:

$$
\int_{\partial \Omega}|d||V|^{2} d \sigma \leq\|d\|_{L_{\infty}(\partial \Omega)}\|V\|_{L_{2}(\partial \Omega)}^{2} \leq B_{1}^{2}\|d\|_{L_{\infty}(\partial \Omega)}\|V\|_{H^{1}(\Omega)}^{2},
$$

where the constant $B_{1}$ comes from the trace theorem (see Lemma 2.1).

By Lemma 2.2 we get

$$
\int_{\partial \Omega}|d||V|^{2} d \sigma \leq B_{1}^{2} B_{2}^{2}(1+H)^{2}\|d\|_{L_{\infty}(\partial \Omega)}\|\mathbf{D}(V)\|_{L_{2}(\Omega)}^{2} .
$$

The second term is estimated as follows:

$$
\int_{\Omega}\left|v^{2}\right||V||\nabla V| d x \leq B_{2}^{2}(1+H)^{2} S_{0}\|\mathbf{D}(V)\|_{L_{2}(\Omega)}^{2} .
$$

Thus, we get

$$
\|\mathbf{D}(V)\|_{L_{2}(\Omega)}^{2} \leq \frac{B_{2}^{2}(1+H)^{2}}{\nu}\left(B_{1}^{2}\|d\|_{L_{\infty}(\partial \Omega)}+S_{0}\right)\|\mathbf{D}(V)\|_{L_{2}(\Omega)} .
$$


This yields the uniqueness of solutions if

$$
\frac{B_{2}^{2}(1+H)^{2}}{\nu}\left(B_{1}^{2}\|d\|_{L_{\infty}(\partial \Omega)}+S_{0}\right)<1,
$$

which is $(1.39)$.

4. Expansion of the solution. In this section we investigate the structure of the unique solutions. By Theorem B, we restrict our attention to small data.

The first component of the expansion of the velocity will be a vector $\mathcal{D}$, defined as a solution to the problem

$$
\begin{array}{ll}
\operatorname{rot} \mathcal{D}=0 & \text { in } \Omega, \\
\operatorname{div} \mathcal{D}=0 & \text { in } \Omega, \\
n \cdot \mathcal{D}=d & \text { on } \partial \Omega .
\end{array}
$$

Lemma 4.1. Let $d \in W_{\infty}^{1}(\partial \Omega)$. Then there exists a unique solution of problem (4.1) such that $\mathcal{D} \in W_{p}^{1}(\Omega)$ for any $2<p<\infty$ and

$$
\|\mathcal{D}\|_{W_{p}^{1}(\Omega)} \leq c(\Omega, p)\|d\|_{W_{\infty}^{1}(\partial \Omega)} .
$$

In particular, if $0<a<1-2 / p$, then

$$
\|\mathcal{D}\|_{C^{a}(\Omega)} \leq c(\Omega, p)\|d\|_{W_{\infty}^{1}(\partial \Omega)} .
$$

Proof. Since our domain is simply connected, by $(4.1)_{2}$ and the Poincaré Lemma there exists a scalar function (stream function) $\varphi_{0}$ such that

$$
\mathcal{D}=\left(-\partial_{x_{2}} \varphi_{0}, \partial_{x_{1}} \varphi_{0}\right)
$$

By $(4.1)_{3}$ and (4.4) we get

$$
n \cdot \mathcal{D}=\frac{d}{d s} \varphi_{0}=0,
$$

where $s$ is the arc length parameter of the curve $\partial \Omega$. Since $\varphi_{0}$ is defined up to a constant we obtain the elliptic problem

$$
\begin{array}{ll}
\Delta \varphi_{0}=0 & \text { in } \Omega, \\
\left.\varphi_{0}\right|_{\partial \Omega}=b & \text { on } \partial \Omega,
\end{array}
$$

where

$$
b(s)=\int_{s_{0}}^{s} d(t) d t
$$

for a fixed point $s_{0} \in \partial \Omega$ and $b\left(s_{0}\right)=0$.

By the assumptions,

$$
b \in W_{\infty}^{2}(\partial \Omega), \quad\|b\|_{W_{\infty}^{2}(\partial \Omega)} \leq c\|d\|_{W_{\infty}^{1}(\partial \Omega)} .
$$


Problem (4.6) is ill posed in the $L_{\infty}$-space. Therefore we embed $W_{\infty}^{2}(\partial \Omega)$ into $W_{p}^{2}(\partial \Omega)$ for sufficiently large finite $p(2<p<\infty)$. Moreover, to avoid unnecessary complications we embed $W_{p}^{2}(\partial \Omega)$ into the trace space $W_{p}^{2-1 / p}(\partial \Omega)$, i.e.

$$
W_{p}^{2}(\partial \Omega) \subset W_{p}^{2-1 / p}(\partial \Omega), \quad\|b\|_{W_{p}^{2-1 / p}(\partial \Omega)} \leq c\|b\|_{W_{p}^{2}(\partial \Omega)} .
$$

Then the standard theory $[1,2]$ for the Laplace operator (Lemma 2.3) yields

$$
\left\|\varphi_{0}\right\|_{W_{p}^{2}(\Omega)} \leq c(\Omega)\|d\|_{W_{p}^{2-1 / p}(\partial \Omega)} .
$$

Then from definition (4.4) and the imbedding theorems we get (4.2) and (4.3). Lemma 4.1 is proved.

Next, we study the behavior of the vorticity. Linearization of the vorticity problem yields

$$
\begin{array}{ll}
-\nu \Delta \alpha_{0}=\operatorname{rot} F & \text { in } \Omega, \\
\left.\alpha_{0}\right|_{\partial \Omega}=-2 d_{, s} & \text { on } \partial \Omega .
\end{array}
$$

Lemma 4.2. Let $F \in L_{2}(\Omega)$ and $d_{s} \in L_{\infty}(\partial \Omega)$. Then there exists a unique solution of problem (4.11) such that

$$
\alpha_{0} \in L_{\infty}(\Omega)+H_{0}^{1}(\Omega)
$$

and

$$
\left\|\alpha_{0}\right\|_{L_{\infty}(\Omega)+H_{0}^{1}(\Omega)} \leq c\left(\left\|d_{, s}\right\|_{L_{\infty}(\partial \Omega)}+\frac{1}{\nu}\|F\|_{L_{2}(\Omega)}\right) .
$$

Proof. To obtain (4.13) we repeat the method used for (1.26). Multiply $(4.11)_{1}$ by

$$
\left(\alpha_{0}-k^{*}\right)_{+}=\max \left\{\alpha_{0}-k^{*}, 0\right\}
$$

with

$$
k^{*}=\sup _{x \in \partial \Omega}-2 d, s
$$

and integrate over $\Omega$ to get

$$
\left\|\nabla\left(\alpha_{0}-k^{*}\right)_{+}\right\|_{L_{2}(\Omega)} \leq \frac{1}{\nu}\|F\|_{L_{2}(\Omega)} .
$$

The same holds for the negative part of $\alpha_{0}$ with $k_{*}=\inf _{x \in \partial \Omega}-2 d_{, s}$. This way we get (4.13). Lemma 4.2 is proved.

As we see, the term $\alpha_{0}$ gives a new term to the velocity expansion of the same order as $\mathcal{D}$. Hence again we study the velocity problem. Examine the system

$$
\begin{array}{ll}
\operatorname{rot} u_{0}=\alpha_{0} & \text { in } \Omega, \\
\operatorname{div} u_{0}=0 & \text { in } \Omega, \\
n \cdot u_{0}=0 & \text { on } \partial \Omega .
\end{array}
$$


Lemma 4.3. Let $\alpha_{0}$ be as in Lemma 4.2. Then there exists a unique solution to problem (4.17) such that $u_{0} \in C^{a}(\Omega)$ and

$$
\left\|u_{0}\right\|_{C^{a}(\Omega)} \leq c\left\|\alpha_{0}\right\|_{L_{\infty}(\Omega)+H_{0}^{1}(\Omega)} .
$$

To prove the above result it is enough to repeat the proof of Lemma 4.1, remembering that $H_{0}^{1}(\Omega) \subset L_{p}(\Omega)$ for any $p<\infty$ if $\operatorname{dim} \Omega=2$.

We now decompose the velocity using $\mathcal{D}$ and $u_{0}$ as follows:

$$
v=\mathcal{D}+u_{0}+u
$$

and the vorticity as

$$
\alpha=\alpha_{0}+\beta .
$$

Lemma 4.4. Let $\mathcal{D}, u_{0}$ and $\alpha_{0}$ be as in Lemmas 4.1-4.3. Then

$$
\begin{aligned}
\left\|v-\left(\mathcal{D}+u_{0}\right)\right\|_{C^{a}(\Omega)} & \leq S_{2}\left\|\alpha_{0}\right\|_{L_{\infty}(\Omega)+H_{0}^{1}(\Omega)}, \\
\left\|\alpha-\alpha_{0}\right\|_{L_{\infty}(\Omega)+H_{0}^{1}(\Omega)} & \leq S_{2}\left\|\alpha_{0}\right\|_{L_{\infty}(\Omega)+H_{0}^{1}(\Omega)} .
\end{aligned}
$$

Proof. Subtraction of (1.11), (1.14) and (4.11) gives

$$
\begin{array}{ll}
v \cdot \nabla \beta-\nu \Delta \beta=-v \cdot \nabla \alpha_{0} & \text { in } \Omega, \\
\beta=(2 \chi-f / \nu) u \cdot \tau & \text { on } \partial \Omega .
\end{array}
$$

We handle problem (4.22) just as in the uniqueness issue to obtain the bound (4.23) $\quad\|\beta\|_{L_{\infty}(\Omega)+H_{0}^{1}(\Omega)}$

$$
\leq S_{1}\left\|\alpha_{0}\right\|_{L_{\infty}(\Omega)+H_{0}^{1}(\Omega)}+\|2 \chi-f / \nu\|_{L_{\infty}(\partial \Omega)}\|u\|_{C(\Omega)} .
$$

To get information about the velocity, note that the perturbation $u$ satisfies

$$
\begin{array}{ll}
\operatorname{rot} u=\beta & \text { in } \Omega, \\
\operatorname{div} u=0 & \text { in } \Omega, \\
n \cdot u=0 & \text { on } \partial \Omega .
\end{array}
$$

Using Lemma 4.3 we conclude that

$$
\|u\|_{C^{a}(\Omega)} \leq A(\Omega)\|\beta\|_{L_{\infty}(\Omega)+H_{0}^{1}(\Omega)} .
$$

Thus we get

$$
\begin{aligned}
& \|\beta\|_{L_{\infty}(\Omega)+H_{0}^{1}(\Omega)} \\
& \quad \leq\left(1-A(\Omega)\|2 \chi-f / \nu\|_{L_{\infty}(\partial \Omega)}\right)^{-1} S_{1}\left\|\alpha_{0}\right\|_{L_{\infty}(\Omega)+H_{0}^{1}(\Omega)}
\end{aligned}
$$

and

(4.27) $\quad\|u\|_{C^{a}(\Omega)}$

$$
\leq A(\Omega)\left(1-A(\Omega)\|2 \chi-f / \nu\|_{L_{\infty}(\partial \Omega)}\right)^{-1} S_{1}\left\|\alpha_{0}\right\|_{L_{\infty}(\Omega)+H_{0}^{1}(\Omega)} .
$$

Lemma 4.4 is proved. 
Next, we want to construct a higher order approximation of the solutions. Set

$$
\begin{aligned}
& v=v^{k}+u=u^{0}+u^{1}+\cdots+u^{k}+u, \\
& \alpha=\alpha^{k}+\beta=\beta^{0}+\beta^{1}+\cdots+\beta^{k}+\beta,
\end{aligned}
$$

where $u^{0}=\mathcal{D}+u_{0}$ and $\beta^{0}$ is defined by Lemma 4.4 , and $u^{k}$ and $\beta$ for $k \geq 1$ are solutions to the following problem:

$$
\begin{array}{ll}
v^{k-1} \cdot \nabla \beta^{k}-\nu \Delta \beta^{k}=-u^{k-1} \cdot \nabla \alpha^{k-1} & \text { in } \Omega, \\
\beta^{k}=(2 \chi-f / \nu) u^{k} \cdot \tau & \text { on } \partial \Omega, \\
\operatorname{rot} u^{k}=\beta^{k} & \text { in } \Omega, \\
\operatorname{div} u^{k}=0 & \text { in } \Omega, \\
n \cdot u^{k}=0 & \text { on } \partial \Omega .
\end{array}
$$

LEMMA 4.5. The solutions of (4.29) satisfy

$$
\begin{gathered}
\left\|u^{k}\right\|_{C^{a}(\Omega)}+\left\|\beta^{k}\right\|_{L_{\infty}(\Omega)+H_{0}^{1}(\Omega)} \leq \Xi^{k}, \\
\left\|v-v^{k}\right\|_{C^{a}(\Omega)}+\left\|\alpha-\alpha^{k}\right\|_{L_{\infty}(\Omega)+H_{0}^{1}(\Omega)} \leq B \Xi^{k+1} .
\end{gathered}
$$

Proof. Just as for (1.26), we find estimates for $\beta^{k}$ and $v^{k}$ :

$$
\left\|\beta^{k}\right\|_{L_{\infty}(\Omega)+H_{0}^{1}(\Omega)} \leq B\left\|u^{k-1} \alpha^{k-1}\right\|_{L_{2}(\Omega)} \leq \Xi^{k-1} B S_{0} .
$$

Hence

$$
\left\|u^{k}\right\|_{C^{a}(\Omega)} \leq A \Xi^{k-1} B S_{0} .
$$

From (4.31) and (4.32) we deduce (4.30) 1 .

To show bounds on the errors note that summing systems (4.29) over $k=0,1, \ldots, m$ we get

$$
\begin{array}{ll}
v^{m-1} \cdot \nabla \alpha^{m}-\nu \Delta \alpha^{m}=0 & \text { in } \Omega, \\
\alpha^{m}=(2 \chi-f / \nu) v^{m} \cdot \tau-2 d_{, s} & \text { on } \partial \Omega, \\
\operatorname{rot} v^{m}=\alpha^{m} & \text { in } \Omega, \\
\operatorname{div} v^{m}=0 & \text { in } \Omega, \\
n \cdot v^{m}=d & \text { on } \partial \Omega .
\end{array}
$$

Then subtracting (1.11), (1.14) and (1.15) from (4.33) we obtain

$$
\begin{array}{ll}
v^{m-1} \cdot \nabla \beta-\nu \Delta \beta=-u \cdot \nabla \alpha-u^{m} \cdot \nabla \alpha & \text { in } \Omega, \\
\beta=(2 \chi-f / \nu) u \cdot \tau & \text { on } \partial \Omega, \\
\operatorname{rot} u=\beta & \text { in } \Omega, \\
\operatorname{div} u=0 & \text { in } \Omega, \\
n \cdot u=0 & \text { on } \partial \Omega .
\end{array}
$$


Estimating solutions of (4.34) we obtain

$$
\|\beta\|_{L_{\infty}(\Omega)+H_{0}^{1}(\Omega)} \leq\|2 \chi-f / \nu\|_{L_{\infty}(\partial \Omega)}\|u\|_{C(\Omega)}+B\left\|u^{m} \cdot \nabla \alpha\right\|_{L_{2}(\Omega)}
$$

but again

$$
\|u\|_{C(\Omega)} \leq A(\Omega)\|\beta\|_{L_{\infty}(\Omega)+H_{0}^{1}(\Omega)},
$$

which implies (since $A(\Omega)\|2 \chi-f / \nu\|_{L_{\infty}(\partial \Omega)}<1$ )

$$
\|\beta\|_{L_{\infty}(\Omega)+H_{0}^{1}(\Omega)} \leq B\left\|u^{m} \cdot \nabla \alpha\right\|_{L_{2}(\Omega)} .
$$

Thus

$$
\|\beta\|_{L_{\infty}(\Omega)+H_{0}^{1}(\Omega)} \leq B \Xi^{m}\|\nabla \alpha\|_{L_{2}(\Omega)} .
$$

Solving $(4.34)_{3,4,5}$ we get

$$
\|u\|_{C^{a}(\Omega)} \leq B \Xi^{m+1} .
$$

Theorem $\mathrm{C}$ is proved.

\section{References}

[1] S. Agmon, A. Douglis and L. Nirenberg, Estimates near the boundary for solutions of elliptic partial differential equations satisfying general boundary conditions I, Comm. Pure Appl. Math. 12 (1959), 623-727.

[2] - , 一, - Estimates near the boundary for solutions of elliptic partial differential equations satisfying general boundary conditions II, ibid. 17 (1964), 35-92.

[3] A. V. Fursikov, M. D. Gunzburger and L. S. Hou, Inhomogeneous boundary value problems for the three-dimensional evolutionary Navier-Stokes equations, J. Math. Fluid Mech. 4 (2002), 45-75.

[4] G. P. Galdi, An Introduction to the Mathematical Theory of the Navier-Stokes Equations, Springer, 1994.

[5] P. B. Mucha, The Navier-Stokes equations and the maximum principle, Int. Math. Res. Not. 2004, no. 67, 3585-3605.

[6] K. Pileckas, Recent advances in the theory of Navier-Stokes equations in domains with non-compact boundaries, in: Mathematical Theory in Fluid Mechanics (Paseky, 1995), Pitman Res. Notes Math. Ser. 354, Longman, 1996, 30-85.

[7] R. Temam, Navier-Stokes Equations, North-Holland, Amsterdam, 1977.

Institute of Applied Mathematics and Mechanics

Warsaw University

Banacha 2

02-097 Warszawa, Poland

E-mail: mucha@hydra.mimuw.edu.pl

Received on 5.6.2003;

revised version on 30.1.2004 